\title{
nephroulm fachtagung \\ Nephro Fachtagung Ulm \\ 10.-11.03.2017 Hotel Seligweiler
}

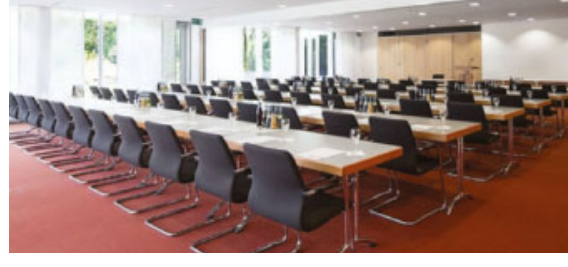

Zum siebten Mal möchten wir, die staatlich anerkannte Nephrologische Weiterbildungsstätte Ulm (www.wbs-ulm.de) in Kooperation mit der AfnP e. V. (www.afnp. de) und dem Georg Thieme Verlag (www. thieme.de) Sie herzlich zur Nephro Fachtagung Ulm in die I(i)ebenswerte Doppelstand Ulm/Neu-Ulm einladen. Mit 170000 Einwohnern zählt sie zu den 8 größten Städten Süddeutschlands. Sie finden hier historisches Ambiente und moderne lebendige Kulturszene nebeneinander. Durch die verkehrstechnisch zentrale Lage in Süddeutschland an der Autobahn A8 (Stuttgart-München) und A7 (Würzburg -Kempten) ist sie für alle gut erreichbar. Auch mit der Bahn ist die Doppelstadt gut angebunden. Ebenso ermöglichen 3 Flughäfen (Stuttgart $70 \mathrm{~km}$, Memmingen $40 \mathrm{~km}$ und München $140 \mathrm{~km}$ ) eine schnelle und komfortable Anreise.

Das multidisziplinäre Programm mit namhaften und anerkannten Referenten wird Sie ansprechen. Parallel zu den Vorträgen bieten Firmen Workshops an. Wir wünschen uns einen anregenden Gedankenaustausch mit unseren Fachreferenten und den Teilnehmern. Ein Blick über den Tellerrand hinaus hat noch nie geschadet.

\section{Termin:}

Freitag, 10.03.2017: 09:00 bis ca. 16:30 Uhr Samstag, 11.03.2017: 08:30 bis ca. 16:00Uhr

\section{Zielgruppe:}

Die Veranstaltung richtet an alle in der Nephrologie tätigen Berufsgruppen wie Pflegekräfte, medizinische Fachangestellte, Diätassistenten, Techniker und Ârzte.

\section{Fortbildungspunkte:}

Beruflich Pflegende erhalten für die Teilnahme Registrierungspunkte für Pflegekräfte, Ärzte erhalten CME-Punkte.

\section{Vorläufiges Programm (Stand: 13.10.2016)}

Unsere Programme gestaltet der Programmbeirat und wählt Referenten aus, die nicht im finanziellen Interessenskonflikt stehen. Wichtig ist für uns die neutrale Information bei unseren Veranstaltungen. Haben Sie ein Thema, das in unser Programm passt und möchten Sie gerne als Referent kommen? Sie können uns auch gerne ein Thema oder Referenten vorschlagen. Teilnehmer können die Vorträge auf www.nephro-ulm.de herunterladen. Ein gemeinsames Abendprogramm im Hotel Seligweiler am Freitag, den 10.03.2017, ab 18:00 Uhr ist in Vorbereitung.

- Geschlechterspezifische Kommunikationsmuster - Ein kurzer Überblick (Oliver Keifert, Bernstadt)

- Frauen in Gesundheitsberufen (Marion Bundschu, Ulm)

- Gendermedizin (Prof. Marion Haubitz, Fulda)

- Nierenersatztherapie in der Intensivtherapie (Dr. Severin Lempp, N. N.; Stuttgart)

- Gerinnung - Heparin-induced thrombopenia (HIT) (Dr. Joachim Buchholz, Coburg)

- Wie kann die Patientenkooperation aus ärztlicher und pflegerischer Sicht verbessert werden? (Prof. Andreas Vychytil, Alois
Tagungsort und Übernachtung:

Hotel und Rasthaus Seligweiler GmbH \& Co KG, Seligweiler 1, 89081 Ulm-Seligweiler, det sich im nahen Umfeld der Innenstadt von Ulm $(8 \mathrm{~km})$, mit sehr guter Verkehrsten wir eine attraktive Übernachtungspauschale vereinbaren (bitte bei der Codewort „Nephro Tagung“ angeben).

Anreise und Parkmöglichkeiten:

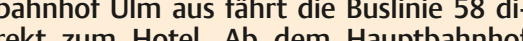
können Sie im Vorfeld unter der Ruf07345/9699-0 ein Sammeltax in sich links in Richtung Hauptpostamt hal列 (ie folgende Anschrift: Ort: LangenDirekt am Hotel Seligweiler stehen kosTagungskosten: Tage nur 90 Euro. Die Tageskarte kostet An der Kasse ist und nicht übertragbar.

Vorteil für AfnP-Mitglieder:

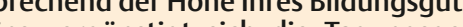
vergünstigt sich die Tagungsgebühr. Bitte bringen Sie Ihren Bildungsgutschein für 2017, Ihren Mitgliedsausweis und Ihren Personalausweis mit. Werden Sie Mitglied und nutzen Sie die zahlreichen Vorteile. Einen Mitgliedsantrag finden Sie auf www.afnp.de.

Anmeldung:

Eine schriftliche Anmeldung und Nutzung der Frühregistrierung ist bis zum 15.02.2017 ist möglich (Vorabüberweisung bis 22.02.2017). Nach dieser Zeit können Sie sich gerne weiterhin ohne Nutzung des Frühbucherrabatts anmelden. Oder Sie kommen unangemeldet zur Veranstaltung und zahlen in bar die Tagungsgebühr. Bitte nutzen Sie das Anmeldeformular auf www.nephro-ulm.de und melden Sie sich schriftlich per Fax, Post oder per Mail (info@nephro-ulm.de) an. Sie erhalten per E-Mail eine Anmeldebestätigung. Mit der Anmeldebestätigung und Ihrem Überweisungsformular kommen Sie an die Kasse.

\section{Ohne Anmeldung:}

Auch ohne Anmeldung können Sie zur Tagung kommen. Bitte planen Sie dann entsprechende Wartezeit an der Kasse ein.

Öffnungszeiten der Kasse:

Donnerstag, den 09.03.2017: ca. 17:0018:00Uhr

Freitag, den 10.03.2017: 07:30-10:00 Uhr Samstag, den 11.03.2017:08:00-10:00Uhr Industrieausstellung:

Produktinformationen können platziert und Gespräche mit den Teilnehmer in den Pausen geführt werden. Bitte wenden Sie sich für weitere Informationen und die Buchung von Tischflächen an das Tagungsbüro.

Tagungsbüro und weitere Informationen: Nephro Fachtagung Ulm, Marion Bundschu, Käppelesweg 8, 89129 Langenau, Tel.: 07345/22933, E-Mail: info@nephroulm.de, Internet: www.nephro-ulm.de

\section{Ullmann; Wien (Österreich))}

- Natriumprofile - Theorie und praktische Anwendung (Carmen Reißenweber, Spardorf)

- PD bei Diabetes mellitus - Ärztliche und pflegerische Sicht (Prof. Andreas Vychytil, Alois Ullmann; Wien (Österreich))

- Schwangerschaft und Nierenkrankheiten (Prof. Sylvia Stracke, Greifswald)

- Konservative Therapie bei geriatrischen Patienten statt Nierenersatzverfahren (OMR Dr. sc. med. Wilfried Dschietzig, Cottbus)

- Post-Transplantations-Diabetes mellitus (Dr. Martina Guthoff, Tübingen)

- Gewalt in der Pflege (Michael Metzger, München)

- Blocklösung (Prof. Frieder Keller, Adalbert Natterer; Ulm)

- Management des Dialysekatheters (Udo Irmer, Markelsheim)

- Workshop: Update - Ernährungstherapie in der Nephrologie (Irmgard Landthaler, München)

- Workshop: Ultraschallgestützte Gefäßpunktion (Hans-Martin Schröder, Neuwied)

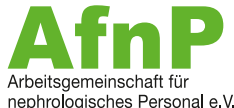

WBS nephrologisches Personal e.V. 